\title{
Residual deformity of the spinal canal in patients with traumatic paraplegia and secondary changes of the spinal cord
}

\author{
R Abel ${ }^{1}$, HJ Gerner ${ }^{2}, \mathrm{C} \mathrm{Smit}^{2}$ and T Meiners ${ }^{3}$ \\ ${ }^{1}$ Orthopädische Klinik, Universität Düsseldorf; ${ }^{2}$ Stiftung Orthopädische Universitätsklinik, Heidelberg and \\ ${ }^{3}$ Werner Wicker Klinik, Bad Wildungen, Germany
}

Introduction: The effect of spinal deformity with posttraumatic kyphosis and stenosis of the spinal canal in producing secondary changes of the spinal cord has been discussed for quite some time. Since the advent of magnetic resonance imaging (MRI), $20-40 \%$ of patients with posttraumatic paraplegia are found to develop hydromyelia.

Purpose of our study: To evaluate the influence of residual spinal deformity, defined by the extent of the posttraumatic kyphosis and stenosis, in the development of posttraumatic hydromyelia.

Material and methods: Two hundred and seven cases of traumatic paraplegia with MRI follow-up were reviewed retrospectively. A minimum of 3 years duration between trauma and MRI study was required (mean 10.6 years [3.2-38.3]). For statistical analysis two groups of patients were formed: with hydromyelia and without hydromyelia. After healing of the fracture, the extent of the kyphosis and stenosis, as well as the characteristics of the paraplegia were noted.

Results: We found that 53 patients had hydromyelia. A highly significant correlation was found for the extent of spinal stenosis and the amount of kyphosis. Cluster analysis indicated that patients with more than 15 degrees of posttraumatic kyphosis and more than $25 \%$ of stenosis were twice as likely to develop hydromyelia. The level of the lesion and the remaining neurological function was not proven to have any influence towards the development of hydromyelia.

Conclusions: These results support the idea that chronic mechanical stress to the spinal cord increases the risk for the development of hydromyelia. Surgical reconstruction should be considered for all patients to prevent secondary changes of the spinal cord.

Keywords: spinal cord injury; post traumatic deformity; hydromyelia

\section{Introduction}

Traumatic paraplegia used to be a fatal injury. Patients surviving trauma died from infected bedsores, pneumonia, renal failure and other sequelae. When long-term survival became the rule, not the exception, a percentage of these survivors showed secondary deterioration of their neurological situation. The formation of posttraumatic cysts in the spinal cord soon became a focus of interest. Initially, the presence of these cysts could only be assumed when a widening of the cervical cord could be visualized in myelograms, later the use of delayed CTscans offered somewhat better diagnostic possibilities and authors ${ }^{1,2}$ reported that up to $3 \%$ of these patients would develop intramedullary cysts. With the advent of the MRI this condition could be demonstrated in $20 \%-$ $40 \%$ of all patients. ${ }^{3-5}$

There is still no common nomenclature for intramedullary cysts, nor has any fully accepted

Correspondence: $\operatorname{Dr} \mathrm{R}$ Abel, Orthopädische Universitätsklinik, Universität Düsseldorf, Moorenstr. 5, 40225 Düsseldorf, Germany model of the pathophysiology been established. It seems likely that some kind of mechanical stress to the cord, namely longitudinal tethering, may be a key factor in their development. ${ }^{6-8}$.

The purpose of this study is to evaluate the influence of the extent of stenosis and posttraumatic kyphosis in the development of hydromyelia.

\section{Materials and methods}

We selected all patients with posttraumatic paraplegia who had been subject to a MRI scan a minimum of 3 years after the injury and were treated in the Werner Wicker Klinik between 1985 and 1995. The clinical records, imaging studies and clinical findings at the time of the MRI were reviewed.

Standard demographic data as well as the exact level of the lesion, additional injuries, changes in the clinical presentation of the paraplegia, and surgical procedures were noted. We included all patients where images as well as sufficient chart documentation was 
available. Two hundred and seven patients, 61 of them female and 146 male met these criteria. The average age at the time of the accident was 28.7 years $(1-68.2$ years). At the time of the MRI study the average age was 39.2 years $(6.2-74.5$ years). We always used the most recent available MRI study. The average time interval between accident and MRI study was 10.4 years, with a range of $3.2-38.37$ years. Reports of clinical follow up were available between 3, 1 and 39.9 years, with an average of 12.7 years.

Indication for MRI in 108 cases were changes of the neurologic situation. A change in the neurological situation was defined as any worsening of the neurologic level (sensory or motor function), including changes of bladder function and otherwise unexplained changes of the type of spasticity. Eightythree patients were referred for MRI because of pain, but for 16 patients, the indication for MRI remained unclear.

One-hundred and thirty-four patients suffered from paraplegia and 73 from tetraplegia (Table 1).

We recorded the presence or absence of additional injuries of the extremities (66 patients), abdomen (12 patients), chest (34 patients), traumatic brain injuries (41 patients) and multilevel spine injuries (eight patients).

Surgery related to spinal injury was classified: no surgery at all (105 patients), posterior decompression only (13 patients), posterior decompression and internal fixation (48 patients), anterior approach only (34 patients), anterior decompression, and posterior internal fixation (seven patients).

The MRI films were read by one trained clinician. The residual deformity in the sagittal plane was measured as the angle between the cranial and caudal margin of the vertebra involved in the trauma. The angle was measured twice and the average of these measurements was used.

The degree of stenosis was scaled to one quarter, half, three-quarters and complete obstruction of the spinal canal. The median sagittal scan was used to determine the location of the maximum stenosis. Appropriate axial scans and scans through the next intact spinal segment cranial to this location were used for the measurement. The degree of stenosis was quantified on an ordinal scale from $0-3$.

The scans were reevaluated for the presence and type of intramedullary cysts. Every cyst was classified into a

Table 1 Distribution of patients according to the Frankel scale

\begin{tabular}{lc}
\hline Frankel scale & $\begin{array}{c}\text { Number of } \\
\text { patients }\end{array}$ \\
\hline 'A' (no motor or sensory function) & 94 \\
'B' (no motor but some sensory function) & 27 \\
'C' (motor function without functional value) & 79 \\
'D' (motor function with functional value) & 7 \\
'E' no motor or sensory deficit & 0 \\
\hline
\end{tabular}

group of cysts that did not exceed the margins of the vertebra involved in the trauma or into the group of cysts that did not exceed these margins.

This differentiation was done because there are different types of intramedullary cysts. Some can be described as remainders of the necrosis taking place at the fracture site (sometimes called 'primary cysts'), and hydromyelia, which extends beyond the location of the injury to the spinal cord in a cranial or distal direction. Whereas the first type of cyst may be considered as a form of scarring with no clinical significance, hydromyelia is supposed to cause additional loss of motor and sensory function due to the intracavity pressure.

There is no clear-cut MRI finding allowing discrimination between these two types of cysts. We considered all cysts that did exceed the upper or lower margins of the vertebra involved in the fracture as hydromyelia (Figure 1), all local, fracture site only cysts were regarded as necrosis (Figure 2).

Statistical advice was given by the Department of Biomedical Mathematics of Heinrich Heine Universität, Düsseldorf. A $P$-level of less than 0.01 was considered highly significant.

\section{Results}

We found cystic formations in 60 patients, $53(25 \%)$ patients had a finding consistent with hydromyelia and seven $(3 \%)$ a cystic lesion meeting the criteria for necrosis. In $147(72 \%)$ patients, no sign of any cystic change was seen.

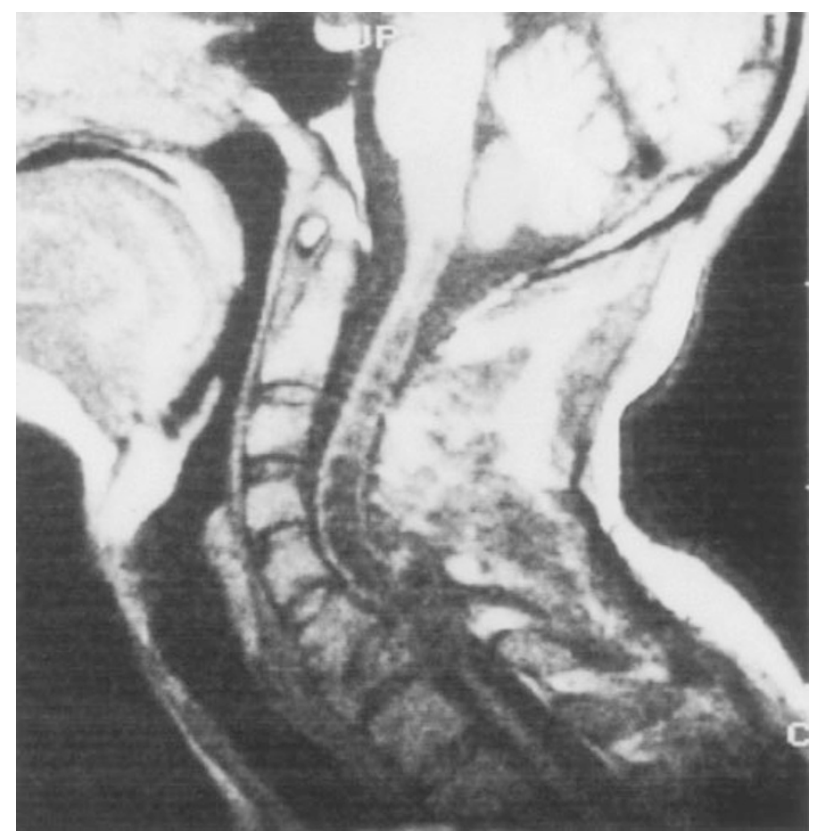

Figure 1 Cervical hydromyelia extending from the fracture site $\mathrm{C} 6 / 7$ to $\mathrm{C} 1$. The spinal cord is dilated and has a central area of hypointensity 
Forty $(75.5 \%)$ of the patients with hydromyelia and $68(44 \%)$ patients without hydromyelia had changes of their neurologic situation.

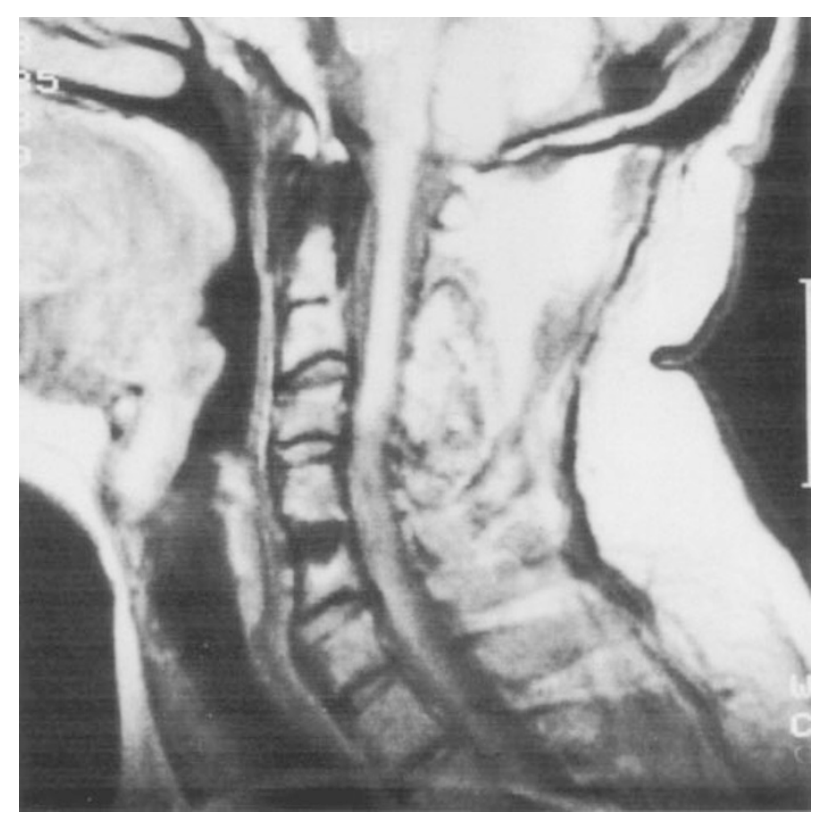

Figure 2 Hypointensive changes within the cervical spinal cord at the level of the fracture $(\mathrm{C} 4 / 5)$. Variable width of the spinal cord in this area but no sign of dilatation
One-hundred, or $48.3 \%$ of our patients had a residual stenosis of the canal of less than $25 \%$ and two patients more than $75 \%$. Seventy-two patients $(34.8 \%)$ had less than 5 degrees of posttraumatic kyphosis. Eighty-five patients have a residual kyphosis between 10 and 35 degrees (Figure 3 ).

There is some, but not very strong, positive relationship between kyphotic deformity and stenosis (kyphosis vs stenosis: stenosis $=0.42+0.18 *$ kyphosis; $r=0.36$ ).

We grouped the patients into those with hydromyelia (Group 1; 53 patients) and those with no hydromyelia (Group 2; 154 patients) according to our definition as given above. When we compared these two groups regarding the posttraumatic angulation, we found a mean angle of kyphosis of 20.53 degrees (range 20-60 degrees; SD 15.45) for Group 1, and 13.90 degrees (range 0-50 degrees; SD 14.16) for Group 2. This finding is highly significant $(t$-test; $P<0.01)$.

Our findings regarding the degree of stenosis of the spinal canal are summarized in Table 2. Patients in the group without hydromyelia generally had a lesser degree of stenosis than the patients with hydromyelia. This finding proved to be highly significant statistically (4 by 2 Table, Pearson chi-square 13.08, $P<0.01$ ).

We did not find differences between patients with and without hydromyelia regarding

- additional injuries

- age at the time of the accident

\section{stenosis}

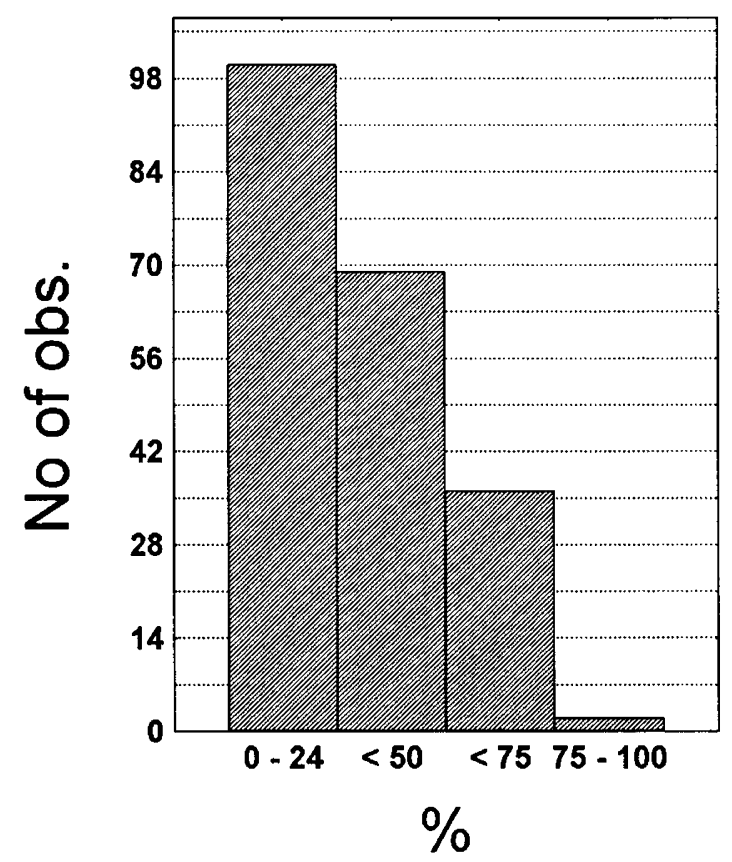

\section{kyphosis}

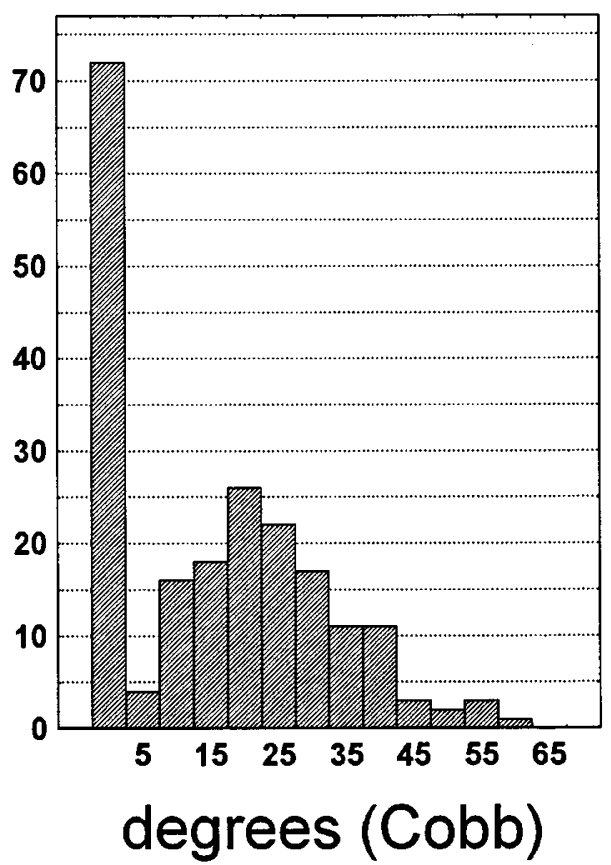

Figure 3 Degree of posttraumatic stenosis and kyposis. This Figure demonstrates the results for 207 patients 
- $\operatorname{sex}$

- level and type of lesion

Concerning the initial management of the fracture, the group which was treated conservatively had the highest incidence of hydromyelia (36 of 105 patients). A lower incidence of hydromyelia was found in the other groups which manifest posterior decompression only (1 of 13 patients), posterior decompression and stabilization ( 9 of 48 patients), anterior surgery only (6 of 36 patients), and anterior and posterior approach (1 of 7 patients). Using a stub and banner Fig to test the statistical significance of this finding, we compared patients with surgical management and no hydromyelia $(n=85)$, surgical management and hydromyelia $(n=17)$, no surgery and no hydromyelia $(n=69)$ and no surgery and hydromyelia $(n=36)$. This result is highly significant $(P<0.01$; Pearson chi-square 8.43$)$. We also compared the average degree of posttraumatic kyphosis, 19.69 degrees for patients without surgical fracture management and 11.42 degrees for patients with surgical fracture management. This finding is also highly significant ( $t$-test, $P<0.01)$. Patients with surgical management tended to have a lesser degree of residual stenosis of the spinal canal (Table 3 ). The finding is highly significant statistically $(P<0.01$; Pearson Chi Square 19.36).

To determine the influence of stenosis and posttraumatic kyphosis a logit model was used. It shows that the probability of developing hydromyelia increases with degree of kyphosis and stenosis. This model does not indicate any abrupt change in probability (Figure 4). It gives no indication of a 'watershed' value, where the incidence of hydromyelia increases sharply, therefore, we used cluster analysis

Table 2 Stenosis of the spinal canal and hydromyelia

\begin{tabular}{lccccc}
\hline & $\begin{array}{c}0-25 \% \\
\text { stenosis }\end{array}$ & $\begin{array}{c}25-50 \% \\
\text { stenosis }\end{array}$ & $\begin{array}{c}50-75 \% \\
\text { stenosis }\end{array}$ & $\begin{array}{c}75-100 \% \\
\text { stenosis }\end{array}$ & \\
\hline Group 1 & 16 & 21 & 16 & 0 & 53 \\
Hydromyelia & $(30.2 \%)$ & $(39.6 \%)$ & $(30.2 \%)$ & $(0 \%)$ & \\
Group 2 & 84 & 48 & 20 & 2 & 154 \\
Hydromyelia & $(54.5 \%)$ & $(31.2 \%)$ & $(13.0 \%)$ & $(1.3 \%)$ & \\
& 100 & 69 & 36 & 2 & 207 \\
\hline
\end{tabular}

Table 3 Stenosis of the spinal canal and surgical management

\begin{tabular}{lccccc}
\hline & $\begin{array}{c}0-25 \% \\
\text { stenosis }\end{array}$ & $\begin{array}{c}25-50 \% \\
\text { stenosis }\end{array}$ & $\begin{array}{c}50-75 \% \\
\text { stenosis }\end{array}$ & $\begin{array}{c}75-100 \% \\
\text { stenosis }\end{array}$ & \\
\hline Group 1 & 36 & 41 & 26 & 2 & 105 \\
Surgery no & $(34.3 \%)$ & $(39.0 \%)$ & $(24.8 \%)$ & $(1.9 \%)$ & \\
Group 2 & 64 & 28 & 10 & 0 & 102 \\
Surgery yes & $(62.7 \%)$ & $(27.5 \%)$ & $(9.8 \%)$ & $(0.0 \%)$ & \\
& 100 & 69 & 36 & 2 & 207 \\
\hline
\end{tabular}

to group our patients. Two clusters could be identified (Figure 5). They indicate that patients with less than 15 degrees of kyphosis and not more than $25 \%$ of posttraumatic stenosis were about half as likely $(17.8 \%$ vs $31.2 \%)$ to develop hydromyelia as patients with a greater extent of the residual deformity. Figure 6 demonstrates the incidence of hydromyelia and a scatter plot of stenosis $v s$ kyphosis in each cluster.

\section{Discussion}

The etiology of the formation of intramedullary cysts is uncertain. There are studies which suggest that chronic mechanical stress to the spinal cord may at least promote the development of hydromyelia. Some authors have noted the following, all of which may play a role in pathogenesis; local arachnoiditis at the fracture site, ${ }^{6,7}$ stretching or tethering of the cord, ${ }^{8}$ or increased intramedullary pressure. ${ }^{9}$ This led us to look for factors that lead to chronic, abnormal,

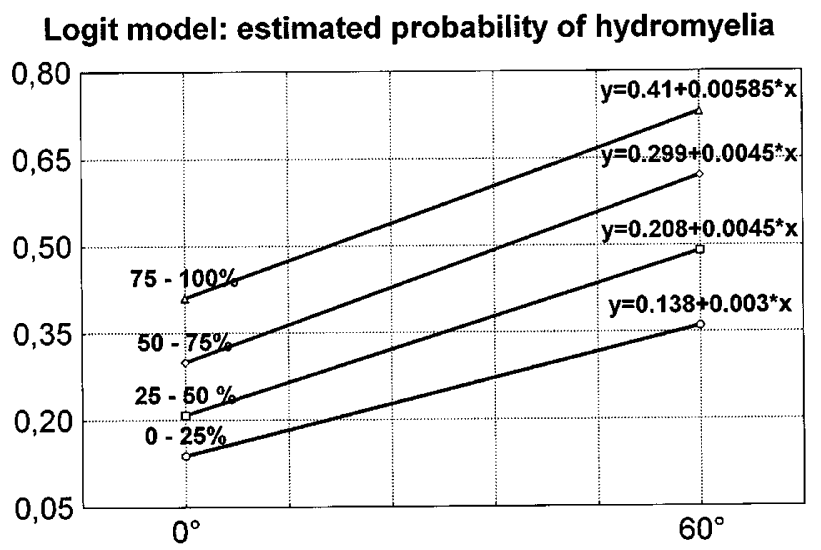

Figure 4 The Logit model shows the increase of the probability for the development of a posttraumatic hydromyelia with increasing kyphosis and stenosis

Tree Diagram for 206 Cases Weighted pair-group average; Euclidean distances

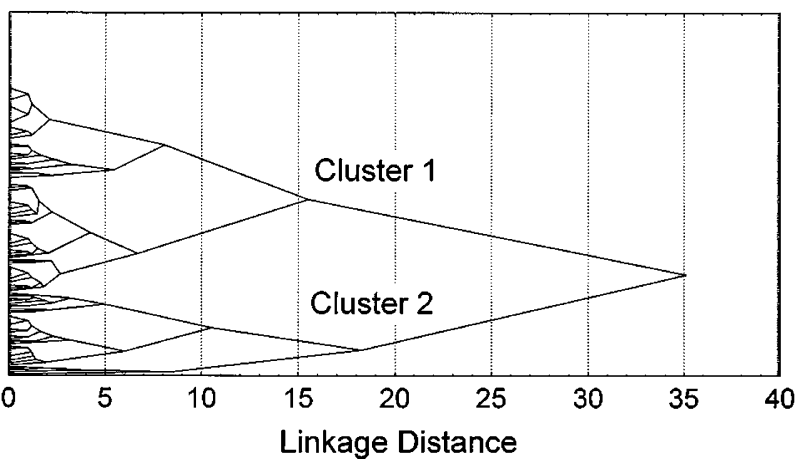

Figure 5 Tree diagram as a graphical representation of the cluster analysis performed for our database 


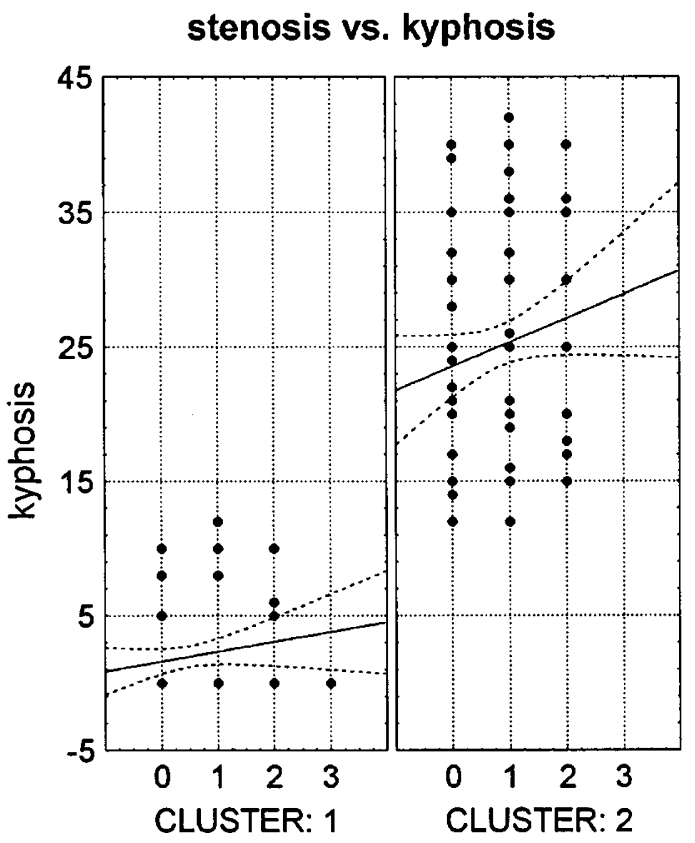

stenosis

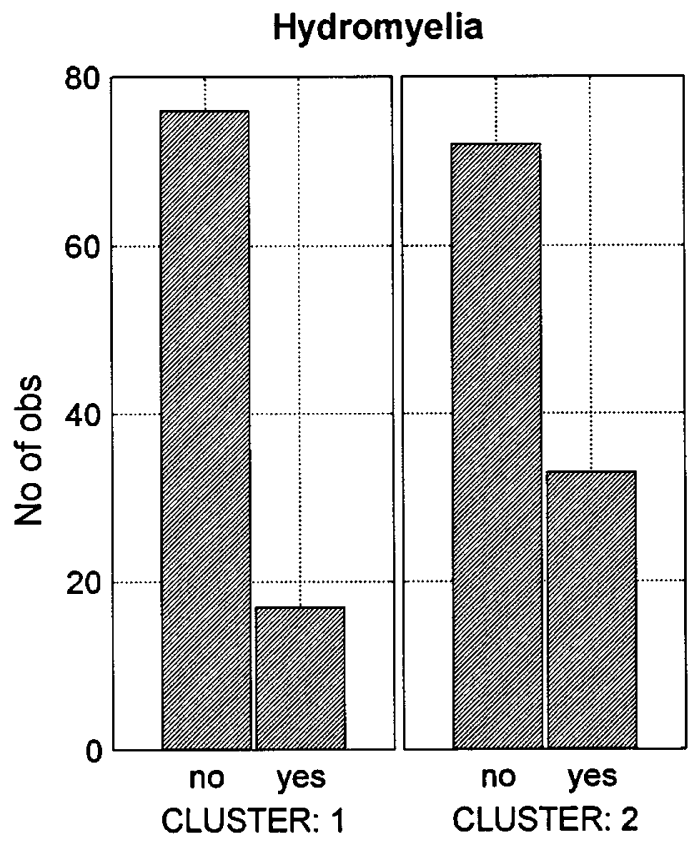

Figure 6 Findings for stenosis and kyphosis in Clusters 1 and 2. The risk for posttraumatic hydromyelia increased from $17.8 \%$ (Cluster 1) to $31.2 \%$ (Cluster 2)

mechanical stress to the spinal cord and may induce localized arachnoiditis. Our hypothesis was that major causes would be residual deformities such as, kyphotic angulations and stenosis of the canal. Obviously, there are other factors such as local adhesions following hematoma or dural lacerations. It has been reported that the reconstruction of the subarachnoid space is a valid option ${ }^{10}$ for the surgical treatment of hydromyelias in order to reestablish free pathways for CSF flow and prevent the recurrence of hydromyelia.

We assumed that we would find more patients with hydromyelia in a population with a higher degree of residual deformity. To test this assumption, we designed our study to look for a correlation between the degree of stenosis, kyphotic angulation, and the development of intramedullary cysts.

According to our results there is a highly significant positive correlation. Since we could not define a 'watershed' value of degree of kyphosis and occlusion of the canal with a logit model analysis, we performed a cluster analysis of our dataset. We found that patients with less than 15 degrees of kyphosis and less than $25 \%$ of posttraumatic stenosis are half as likely to develop hydromyelia than patients with residual deformity exceeding these values. Cluster analysis is a descriptive analysis of a given sample and it is not possible to extrapolate the results for random samples. Therefore, there has to be further investigation as to whether this finding is present in other patient populations. It fits well into this picture that patients who have had surgery to reconstruct the canal tend to develop less frequent hydromyelia than patients without surgical reconstruction of the spine.

The clinical importance of our results lies in the fact that the indication to reconstruct the spine has been based solely on the idea to stabilize the fracture to allow for early mobilization. There has been no proof that neurologic recovery after surgical management is better than with conservative treatment. ${ }^{11,12}$

However, the positive results of secondary reconstruction of the subarachnoid space in cases of posttraumatic hydromyelia ${ }^{10}$ fall in line with our findings.

The results of our study suggest that the prevention of secondary neurologic deterioration should be added to the list of indications for reconstructive spine surgery in posttraumatic paraplegia. Every attempt should be made to reconstruct the canal, including anterior decompression, even if posterior fixation alone would lead to stable healing of the fracture, but result in an undesirable residual deformity.

\section{Acknowledgements}

We thank Mrs Sherill Marciano, Motion Analysis Laboratory, Children's Hospital, San Diego, USA, for her help in the preparation of this manuscript. 


\section{References}

1 Anton HA, Schweigel JF. Posttraumatic syringomyelia: The British Columbia experience. Spine 1986; 11: 865-868.

2 Umbach I, Heilporn A. Post-spinal cord injury syringomyelia. Paraplegia 1991; 29: 219-221.

3 Curati WL, Kingsley DPE, Moseley IF. MRI in chronic spinal trauma. Neuroradiology 1992; 35: 30-35.

4 Wacke HA, et al. Posttraumatic spinal cord cysts evaluated by MRI. Paraplegia 1991; 29: 607-612.

5 Wang $\mathrm{G}$ et al. A clinical magnetic resonance study of the traumatized spinal cord. More than 20 years following injury. Paraplegia 1996; 34: $65-81$.

6 Hida K et al. Posttraumatic Syringomyelia: It's characteristic magnetic resonance imaging findings and surgical management. Neurosurgery 1994; 5: 886-890.
7 Cho KH et al. Experimental model of posttraumatic syringomyelia: The role of adhesive arachnoititis in syrinx formation. Journal of Neurosurgery 1994; 80: $133-139$.

8 Fehlings $\mathrm{MG}$, Tator $\mathrm{CH}$, Illis $\mathrm{L}$, (editors). A review of experimental models of acute spinal cord injury. In: Spinal cord dysfunction and assessment. Oxford: Oxford University Press 1988; pp $30-35$

9 Tachibana S, Kitahara Y, Ida H, Yada K. Spinal cord intramedullary pressure. Spine 1994; 19: 2174-2179.

10 Sgouros S, Williams W. Management and outcome of posttraumatic syringomyelia. J Neurosurg 1996; 85: 197-205.

11 Meinecke FW. Was hat die Wirbelsäulenstabilisation für den Querschnittsgelähmten gebracht? Rehabilitation 1990; 29: 163 168.

12 Gerner HJ, Ingunza W, Kluger P. Therapie und Ergebnisse bei 80 Tetraplegikern-Ein Beispiel interdisziplinärer Kooperation. Orthopäde 1984; 122: 516-517. 